diagnosis, synchronous pathology, referral timelines and reasons for delays.

Results

- 108 patients had a PET-CT for investigation of thoracic malignancy.

- 29 incidental findings were found in 28 patients (28/ 108;26\%); see chart-1.

- 20 patients $(20 / 108 ; 19 \%)$ required further investigation/referral for their synchronous pathology.

- 22 of these 28 patients were ultimately diagnosed and treated for thoracic malignancy.

- Referral to treatment was delayed in 8 patients $(8 / 108 ; 7 \%)$, but only in $1(1 / 108 ; 1 \%)$ as a direct result of management for their synchronous pathology.

Conclusions A high number of patients undergoing PET-CT have synchronous pathology. Compared with previous UK data we found a higher rate of synchronous pathology. This may reflect a higher burden of disease in the North East of England.

Synchronous findings have a limited impact on referral to treatment pathways. However they can result in more investigations and trips to hospital, which may have a psychological impact on patients already going through a stressful life event. Perhaps this should be highlighted at time of decision to perform PET-CT.

\section{P74 FOLLOW-UP OF LUNG CANCER PATIENTS POST SURGERY}

R Aslam, AR Biswas, P Blaxill. Mid Yorkshire NHS Trust, Wakefield, UK

10.1136/thoraxjn-2014-206260.215

Rationale Over 50\% of patients undergoing surgery for lung cancer die from recurrence or a second episode of lung cancer within 5 yrs. ${ }^{1}$ There is little evidence based guidelines regarding the follow up of post surgical resection. Most follow up with physical examination and plain radiographs. Few recommend follow up with CTs. Within Mid Yorkshire NHS trust, patients are followed up post-operatively for 5 years. In the initial 2 years CTs are performed at 3, 12 and 24 months and chest radiographs at 3 to 6 monthly intervals for 5 years. The aim of this study was to assess the benefits of cross sectional imaging.

Methods A retrospective analysis was conducted of 109 patients undergoing surgery for lung cancer within Mid Yorkshire NHS hospital trust between 2009 and 2012.

Results 109 patients were included in total (42\% female). Types of surgery were lobectomy (80\%), wedge resection (10\%) and pneumonectomy (10\%) Recurrence occurred in 37\% of patients (85\% pulmonary).

$60 \%$ of recurrences were adenocarcinomas and $33 \%$ squamous cell carcinomas. The majority of patients were asymptomatic (78\%). Dyspnoea was the most frequent symptom (19\%). The most commonly staged tumour was $1 \mathrm{~B}$ (pT2A 53\%, pN0 $50 \%)$.

$53 \%$ of recurrences were identified at the 3 month post-operative CT, $8 \%$ at 6 months, $28 \%$ at 12 months and $3 \%$ at 24 months. Chest radiographs identified recurrence at $6(3 \%), 9$ (3\%) $18(3 \%)$ and 21 months (3\%). Total mortality within the recurrence group was $25 \%$.

$54 \%$ of patients had treatment with curative intent (surgery; $23 \%$ radiotherapy; $18 \%$ chemotherapy $8 \%$; chemoradiotherapy $5 \%$. 23\% received palliative treatment, chemotherapy/ radiotherapy.
Conclusions There are substantial benefits of imaging in identifying recurrences in cancer patients. The post-operative CT imaging at 3 and 12 months is advantageous as they identified $53 \%$ and $28 \%$ of the recurrences respectively. However, the benefit of regular chest radiographs and surveillance CT at 24 months is questionable as they were less effective.

\section{REFERENCE}

1 Egerman U, Jaeggi $K$, Habicht JM, Perruchoud AP, Dalugen $P$, Soler M. Regular follow-up after curative resection of nonsmall cell lung cancer: a real benefit for patients? Eur Respir J 2002 Mar;19(3):464-8

\section{P75 PROGNOSTIC IMPLICATIONS OF THE MODIFIED GLASGOW PROGNOSTIC SCORE IN EARLY STAGE NON- SMALL CELL LUNG CANCER}

AM MacKenzie, E Johnson, S Tsim, KG Blyth. Department of Respiratory Medicine, Southern General Hospital, Glasgow, UK

\subsection{6/thoraxjn-2014-206260.216}

Introduction and objectives Up to $50 \%$ of patients treated radically for non-small cell lung cancer (NSCLC) subsequently present with metastatic disease. This is despite rigorous caseselection and the use of adjuvant therapies based on clinical and/ or surgical staging. A simple, objective biomarker that identified patients at higher risk of recurrence might facilitate more effective multi-modality radical treatment.

Since inflammation-based biomarkers offer robust prognostication in metastatic NSCLC, we hypothesised that the modified Glasgow Prognostic Score (mGPS), Neutrophil:Lymphocyte Ratio (NLR) and/or Platelet:Lymphocyte Ratio (PLR), measured prior to radical treatment would have utility in this regard.

Methods Utilising a radiology database, we retrospectively identified all patients with Stage I-IIIa NSCLC who underwent

\section{Survival following Surgical Resection for NSCLC}
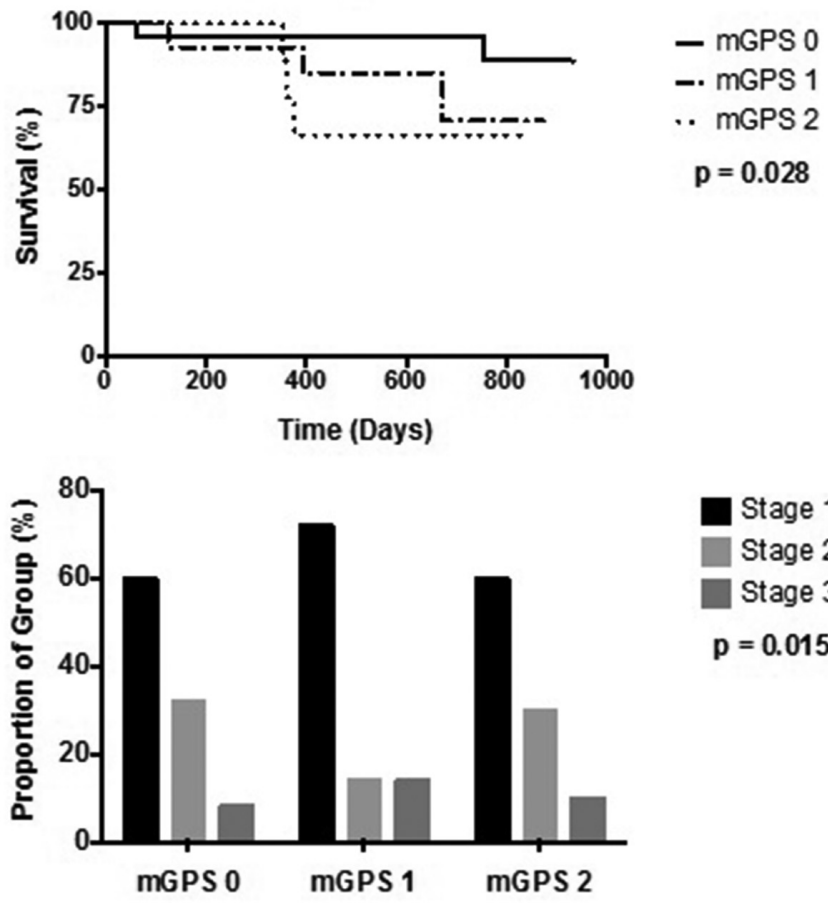

Stage 1

Stage 2

Stage 3

$p=0.015$

Abstract P75 Figure 1 Survival following surgical resection for NSCLC 
radical treatment between August 2011 and August 2012. Electronic records were reviewed and baseline parameters, including blood results were recorded. mGPS (based on CRP and Albumin), NLR and PLR were calculated. All cases were subject to multidisciplinary assessment, detailed staging and 2-year followup. Kaplan-Meier plots were generated for mGPS, NLR, PLR and compared using log-rank for trend and log rank. Differences in mortality were quantified using Hazard Ratios (HR). Differences in stage proportion were compared using the Chi-Square $\mathrm{z}$ test.

Results 97 patients were identified. 44/97 (45\%) were male, mean age $70( \pm 8)$ years. 54/97 (56\%) underwent surgery, 43/97 (44\%) underwent radical RT. NLR and PLR provided no useful prognostic information. In surgical patients only, increasing mGPS was associated with decreasing 2-year survival (see Figure $1(a)$ ), with curve separation occurring 1 year post-resection. Preoperative mGPS 1 and 2 were associated with HR for death of 3.9 (95\% CI $0.8-39.5, \mathrm{p}=0.095)$ and 5.8 (95\% CI 1.38-106, $\mathrm{p}=0.02)$ relative to mGPS 0. There were less Stage I and more Stage II patients in the mGPS 1 group (see Figure 1(b)), mGPS 0 and 2 appeared well matched for stage.

Conclusion These data suggest that pre-operative mGPS may be useful in risk-stratifying patients with early stage NSCLC. The late survival curve separation observed suggests recurrent malignancy rather than post-operative complications are likely to explain this. If confirmed prospectively, integration of mGPS into staging algorithms might allow more effective targeting of adjuvant therapies.

\section{P76 WHEN IS IT SAFE TO DISCHARGE RESECTED STAGE 1A/ 1B NSCLC FROM THE CLINIC?}

${ }^{1} \mathrm{G}$ Kamalatharan, ${ }^{1} \mathrm{CS}$ Moorcroft, ${ }^{2} \mathrm{R}$ Shah, ${ }^{3} \mathrm{SCO}$ Taggart. 'University of Manchester, Manchester, UK; ${ }^{2}$ University Hospital of South Manchester, Manchester, UK; ${ }^{3}$ Salford Royal NHS Foundation Trust, Salford, UK

\subsection{6/thoraxjnl-2014-206260.217}

Introduction We have previously shown that the majority of recurrent disease occurs within the 2 years of lung cancer resection. ${ }^{1}$ Follow-up protocols vary between centres but often involve serial CXR examinations. At Salford we also perform a CT scan at one year after surgery. Given that the prognosis for early stage lung cancer is good, the question arises as to when it's safe to discharge such patients from follow-up? Traditionally this has been set at 5 years.

The Salford Lung Cancer d-base provides comprehensive data on all patients in Salford undergoing surgical resection including outcomes during follow-up. To date, 255 patients have undergone resection of non-small cell lung cancer and the rate of resection is increasing year on year. ${ }^{2}$ This audit sets out to review the data following introduction of routine PET scans to our service in 2005 with a view to providing guidance as to when it might be safe to discontinue regular follow-up of early stage disease.

\begin{tabular}{|c|c|c|c|c|c|}
\hline Stage & Oyr & $1 \mathrm{yr}$ & $2 \mathrm{yr}$ & $3 \mathrm{yr}$ & $4 y$ \\
\hline $1 \mathrm{~A}$ & 23 & 23 & 22 & 22 & $22(96 \%)$ \\
\hline $1 B$ & 20 & 18 & 17 & 17 & $17(85 \%)$ \\
\hline $1 A+1 B$ & 43 & 41 & 39 & 39 & $39(91 \%)$ \\
\hline
\end{tabular}

Methods All patients undergoing surgical resection were first identified from March 2006 to July 2010. Those with a postoperative stage $1 \mathrm{~A}$ or $1 \mathrm{~B}$ disease were then extracted; allowing a 4 year follow up for each patient. Those patients dying within 4 years of surgery from non-cancer and non-lung cancer causes were excluded to produce a selected cohort of patients. 1, 2, 3 and 4 years survival figures were then produced for each category of disease $(1 \mathrm{~A}, 1 \mathrm{~B}$ and $1 \mathrm{~A}+1 \mathrm{~B})$ to observe for any serial changes.

Results A total of 89 patients underwent surgical resection during the study period of which $55(62 \%)$ were $1 \mathrm{~A}$ or $1 \mathrm{~B}$ disease. After exclusions, 43 patients $(23 \times 1 \mathrm{~A}$ and $20 \times 1 \mathrm{~B})$ were available for analysis. As expected, relapse rates were low and occurred in the first 2 years. Survival rates were high but remained stable after 2 years of follow up (see Table). The use of 1 year CT scans detected just 2 relapses.

Conclusions Allowing for the small numbers, the above audit supports a move away from traditional follow-up protocols to discharge alive and well patients with resected early stage disease from the clinic at 2 years. The role of imaging surveillance during the first 2 years requires further exploration.

\section{REFERENCES}

1 Tunney R et al. P195 Temporal Trends and Distribution of Recurrent Disease Following Lung Cancer Surgery and Relationship to Pre-Operative PET-CT. Thorax 2010;65:A159-A160

2 Gornasa M et al. P7 Radical Treatments for Lung Cancer: Impact of Recent Service Improvements. Thorax 2013:68:A77-78

\section{P77 CARCINOMA IN-SITU AT THE BRONCHIAL RESECTION MARGIN - A CASE FOR ROUTINE SURVEILLANCE WITH AUTOFLUORESCENCE BRONCHOSCOPY}

${ }^{1}$ RM Thakrar, ${ }^{1} \mathrm{JM}$ Brown, ${ }^{2} \mathrm{H}$ Apperley, ${ }^{3} \mathrm{M}$ Falzon, ${ }^{4}$ DR Lawrence, ${ }^{3} \mathrm{PJ}$ George, ${ }^{3} \mathrm{~N}$ Navani, ${ }^{1} \mathrm{SM}$ Janes. 'Lungs for Living Research Centre, University College London, UK; ${ }^{2}$ Bristol Medical School, University of Bristol, UK; ${ }^{3}$ Thoracic Medicine, University College London Hospital, UK; ${ }^{4}$ The Heart Hospital, University College London Hospital, UK

\subsection{6/thoraxjnl-2014-206260.218}

Introduction Lung cancer is the leading cause of cancer mortality worldwide, with squamous cell carcinomas commonly arising in the central airways and accounting for nearly $30 \%$ of cases. Progression from normal bronchial epithelium to carcinoma insitu (CIS) has been well described, and is found at the resection margin after lobectomy in up to $2.5 \%$ of cases; however, its fate has not been defined.

Method Cases referred to the autofluoresence bronchoscopy (AFB) surveillance programme at this institution were analysed retrospectively from 1999-2012, for all those shown to have $\mathrm{CIS}$ at the resection margin following surgery for TxN0M0 squamous cell carcinoma. Patients underwent longitudinal assessment of the tracheobronchial tree to (a) confirm CIS at the resection margin and track its fate over time (b) characterise development of other preinvasive lesions.

Results Twenty-two cases were identified with a median interval of 6 months (range 3-9) from surgical resection to first AFB. Thirteen patients (59\%) were confirmed to have CIS on biopsy at the bronchial resection margin during the first AFB. Eleven $(85 \%)$ of these progressed to invasion over a median interval of 37 months (range 4-85). A subgroup of these (5 patients) developed 8 invasive cancers at sites distant to the anastomotic site and 9 patients had $>1$ CIS lesion at a distant site. Two patients (9\%) found to have CIS after initial post-resection AFB, persisted after follow-up of 36-45months. Although no progression 